Conference Paper

\title{
Review of unmanned aircraft system technologies to enable beyond visual line of sight (BVLOS) operations
}

Davies, L., Bolam, R., Vagapov, Y. and Anuchin, A.

This is a paper presented at the 10th International Conference on Electrical Power Drive Systems ICEPDS 2018, Novocherkassk, Russia, 3-6 October 2018.

Copyright of the author(s). Reproduced here with their permission and the permission of the conference organisers.

\section{Recommended citation:}

Davies, L., Bolam, R., Vagapov, Y. and Anuchin, A. (2018) 'Review of unmanned aircraft system technologies to enable beyond visual line of sight (BVLOS) operations'. In Proc. 10th International Conference on Electrical Power Drive Systems ICEPDS 2018, Novocherkassk, Russia, 3-6 October 2018, pp. 1-6. doi: 10.1109/ICEPDS.2018.8571665 


\section{Review of Unmanned Aircraft System Technologies to Enable Beyond Visual Line of Sight (BVLOS) Operations}

\author{
Lee Davies \\ Glyndwr University \\ Wrexham, UK \\ Yuriy Vagapov \\ Glyndwr University \\ Wrexham, UK
}

\author{
Robert Cameron Bolam \\ Glyndwr University \\ Wrexham, UK \\ Alecksey Anuchin \\ Moscow Power Engineering Institute \\ Moscow, Russia
}

\begin{abstract}
The need to develop and deploy Beyond Visual Line of Sight (BVLOS) aerial vehicles has intensified over the last decade. As the demand for Unmanned Aircraft Systems (UAS) has increased, so too has the regulations that surrounds the industry. Strict regulations are currently in place but differ from country to country. Due to these regulations BVLOS innovators have been posed the task of exploring the means of operating flight missions with the UAV out of the sight of the pilot. Autonomous flight capability is not only fundamental to BVLOS operations for UAS but also likely to have a significant impact on the future development of passenger carrying autonomous aircraft. This review explores the technologies that have been developed to date that enable BVLOS applications. BVLOS flight operations have the potential to open a huge area of commercial opportunity however, there remain many concerns about the current capabilities of UAS to detect and avoid manned and unmanned airborne hazards that may pose a significant safety risk.
\end{abstract}

Keywords-drones, unmanned aircraft system, BVLOS, autonomous aircraft

\section{INTRODUCTION}

Accompanying the rapid increase of drone operations over the past few years has been a comparative increase in the regulations governing the industry. The main driver for which has been the safety of societies with respect to their populations, property and environment. This cautious approach has been very successful to date and in the UK, in common with many other European countries, amateur drone operations are only permitted to take place within the Visual Line of Sight (VLOS) of the Remote Pilot. This is commonly interpreted to mean up to $500 \mathrm{~m}$ horizontally and $400 \mathrm{ft}(120 \mathrm{~m})$ vertically. For commercial UAV operators Extended Visual Line of Sight (EVLOS) operations beyond the aforementioned distances may also be permissible. Applications must be submitted to the Civil Aviation Authority (CAA) for EVLOS which include an acceptable safety case and the use of deployed observers. Operations Beyond Visual Line of Sight (BVLOS) may also be permitted if an approved method of aerial separation and collision avoidance is employed or alternatively the flights are made within segregated airspace under Instrument Flying Rules (IFR) and with Air Traffic Control (ATC) clearance [1]. Fig. 1 illustrates VLOS, EVLOS and BVLOS operations.
Recently many national governments have identified UAS as a key economic growth sector for technology and are keen to encourage its development. In June 2017 the Single European Sky Air traffic management Research Joint Undertaking (SESAR Joint Undertaking) released a blueprint aimed at making a strong and dynamic EU drone services market by introducing the concept of "U-Space" a low-level airspace for drone operations [3]. This airspace is intended to be in place by 2019 and extend vertically to $150 \mathrm{~m}$. Drone operations within it are to be safe and automated for BVLOS operations. It has been predicted that the advent of BVLOS operations will herald a new boom in the drone industry [4].

It could be claimed that the first recorded BVLOS UAV mission was carried out by the Austrian army in 1849 with an attack on Venice using hot air balloons filled with explosives. [5] Since then the use of UAVs has increase substantially in both the military and commercial sectors. In the UK, BVLOS flights are more commonly conducted by the military normally under the guidance of the Military Aviation Authority (MAA), but that seems to be about the change as the UK Civil Aviation Authority has granted permission to the Defence Infrastructure Organisation Service Delivery Training (DIO SD TRG), to conduct a BVLOS test at the Salisbury Plain Training area to meet its military requirements [6], [7]. This form of approval for BVLOS flights could be applied to a wide and varied number of government and public applications. There are quite a few scenarios where BVLOS could be executed efficiently and safely such as: package delivery, which has already been tested by Amazon; pipeline inspections that

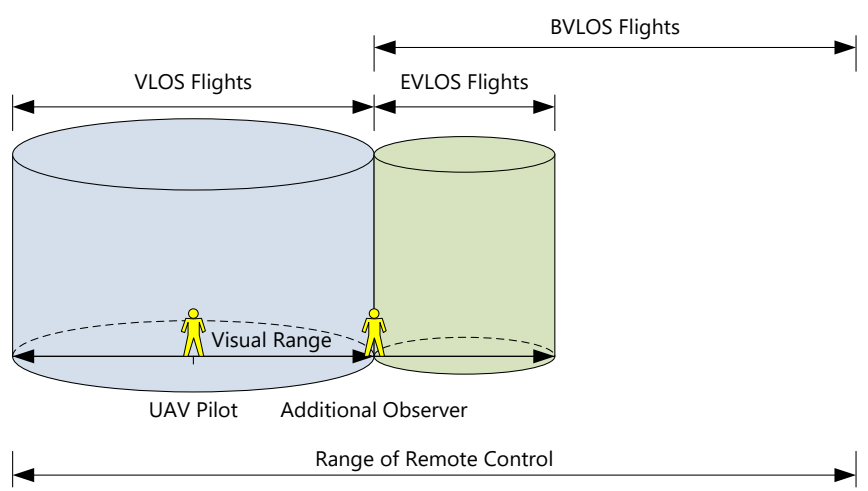

Fig. 1. VLOS, EVLOS and BVLOS illustrated [2]. 


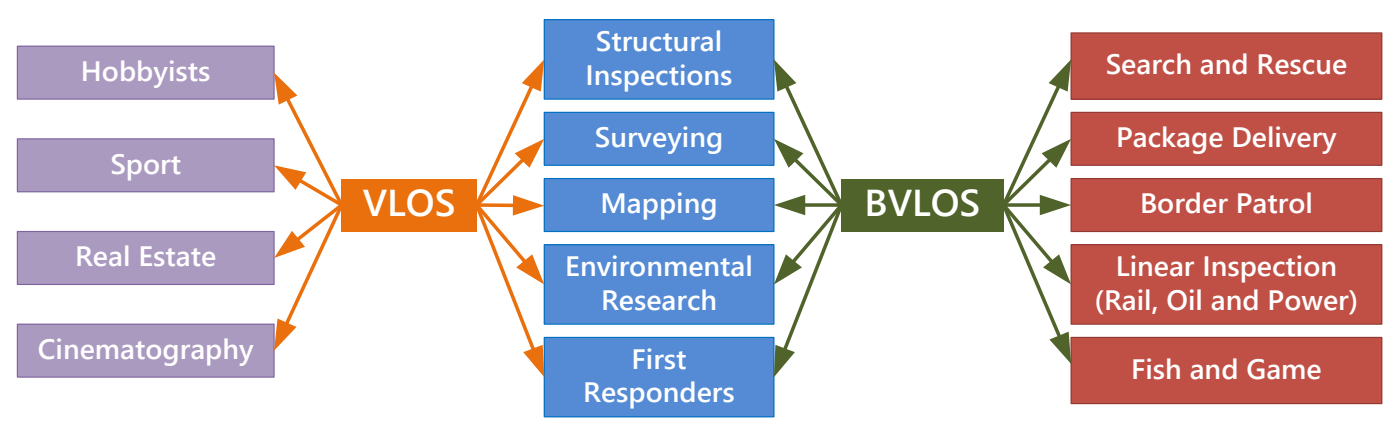

Fig. 2. VLOS and BVLOS mission applications.

stretch over great distances; agriculture; search and rescue; policing and border control etc. [8]-[12]. BVLOS operations can arise from features on the landscape when VLOS mission encounter obstacles such as mountains, dense forests and cities. Fig. 2 demonstrates typical areas of application for VLOS and BVLOS operations.

It is apparent that BVLOS capability is becoming an essential requirement as companies strive to develop autonomous passenger and air freight systems. To achieve safe deployment a UAS will depend on 360-degree radial technologies that allow the vehicle to be aware of its surroundings. The following text reviews the BVLOS situational awareness methodologies and technologies that are currently available or in development.

\section{FIRST PERSON VIEW (FPV) AND DETECT AND AVOID TECHNOLOGIES}

In 2017 Transport Canada issued their unprecedented permission to Ventus Geospatial to perform a BVLOS test. The test was conducted using a Skyranger UAV which reached a distance of 1.4 miles from the operator and was fitted with a camera for a First-Person View (FPV) allowing the live feed to be fed back to a monitored display [13]. For the test run a chase vehicle was also used as a back up to monitor its progress.

FPV is not an uncommon means of technology to use with applications of this nature, although it could be argued that it cannot and should not replace a pilot's own visual range as there is more to BVLOS applications than merely having a visual layout of the surrounding area. Other technologies should also be implemented for a flight plan to be executed safely. According to the reports surrounding the Skyranger test flight, the UAV was not fitted with any detect and avoid technology but in further tests will use Automatic Dependent Surveillance - Broadcast (ADS-B), which is surveillance technology that allows an aircraft to determine its position via satellite navigation and then in turn broadcast it periodically enabling it to be monitored and tracked. [14]. This, however, is not without its problems, such as the security of the UAS. A paper published by Costin and Francillon [15] questioned this lack of security in relation to protocol and practical attacks. The research concluded that there is indeed an inherent insecurity to the commercial grade ADS-B design as it was missing the most basic of security protocols. Taking this into consideration however, one of the most recent ADS-B products has been used for BVLOS operations is the Ping20s which has been successfully used on a UAV. It was used in a successful night and day test which was performed by Australian company V-Tol Aerospace and
UK based RelmaTech [16]. Presently the Ping20s is possibly the world's smallest and affordable Mode S ADS$\mathrm{B}$ transponder and allows UAV's to respond to Mode S radar [17] (Fig. 3). This UAV was also fitted with a GosHawk-II HD sensor and its integrated laser rangefinders can determine exact distance under all environmental conditions. It is also equipped with optical sensors for both night and daytime operations [18]. The need to be able to fly at night is an essential commodity in the drone industry and the development of this technology could pave the way for regulated night missions to become a reality.

There is also an obvious need for a UAV to be aware of its surroundings and aware of other air traffic by using detect and avoid technology. One such technology has been developed and a paper published by Balachandran et al. [19]. The paper explores an approach that enables a multitude of aircraft to coordinate their own manoeuvres. This is achieved by each of the aircraft implicitly agreeing on the region of the airspace that they will be occupying at that time. This in turn has led to the construction of a feedback mechanism that can be executed in real time. The planning of this process assumes that all the aircraft will reside in their own region and it is this assumption that is crucial to ensure that no aircraft are able to occupy the same airspace. Information is shared between the aircraft in relation to when one aircraft speeds up or slows down and will then asses the likelihood of a collision. If an aircraft enters an adjacent zone occupied by another aircraft it will be required to enter a holding pattern until it decides that it is safe to proceed it is therefore much more suited to multirotor UAV's than fixed wing craft. This decisionmaking ability can also serve as a feedback mechanism. The conclusion raised in the paper states that the best

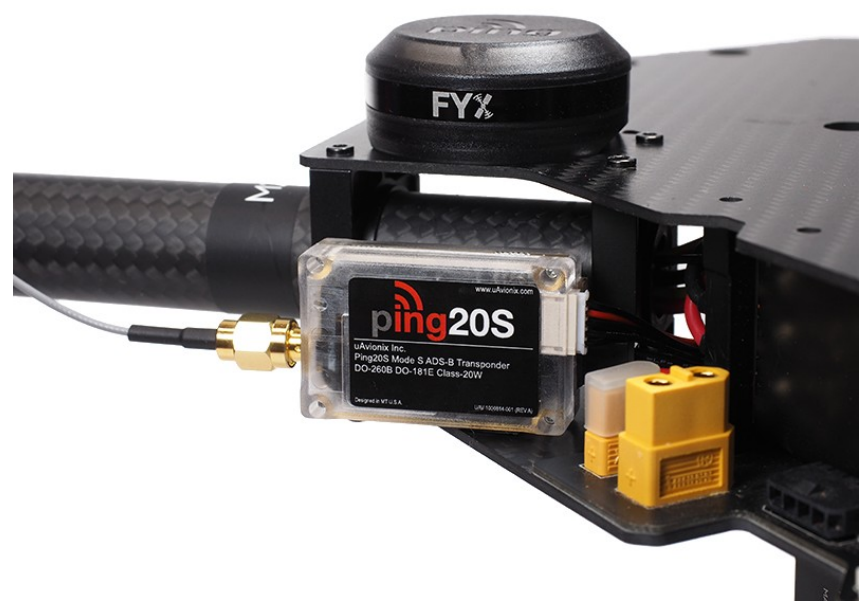

Fig. 3. Ping20s transponder [17]. 
method would be to enforce separation between aircraft by using geo-fencing restraints.

\section{UAS TRAFFIC MANAGEMENT (UTM) SYSTEM}

NASA has been a major contributor to the world of UAS and has explored and developed prototype technologies for a UAS Traffic Management (UTM) system [21]. It is thought that this will enable the integration requirements needed for safe and efficient low altitude applications to be performed [19]. The paper presented by Kopardekar et al. [21] proposed a concept of operations for the UTM model. However, flying drones and small UAV's in a civilian airspace presents its own challenges, for example in the event that there is a need to avoid a forced landing due to collision or due to failings of an aircraft's control system. Their research is based on lessons learned through aviation history and how they can implement that into present day aviation. They believe that it is expected that all UAS will have the ability to operate safely in variable weather conditions and in both controlled and uncontrolled airspace due to the advancement in technologies. All UAS will stay clear of each other as well as manned aircraft and all UAV operators and systems will be required to have up to date awareness of traffic constraints from the ground upwards. The aims of the UTM model is to be flexible in certain areas but vigorously structured in other areas when it is required. It is a riskbased model that is currently aimed at low risk environments and will eventually progress in to higher risk scenarios and environments.

One of the key attributes of NASA's UAS UTM system design is that it would not require any human operators to monitor the vehicles closely at all times. It is proposed that in its fully developed form the system could be further developed to have the following autonomous programming characteristics that include; self-configuration, selfprotection from airborne hazards, land hazards and selfoptimisation during the mission in relation to current and predicted weather conditions. NASA also hopes to deliver two types of UTM systems with one being a portable UTM system that can be transported between areas to support operations. Whilst the second proposed concept would be in constant availability for a geographical area. This would enable the possibility of BVLOS applications to be delivered safely within this area [20]. Working with NASA in this development is Gryphon Sensors who at present have developed a sensor system that detects, identifies and tracks UAS. By using their main product Skylight, it provides an integrated picture consisting of radar for long range detection, spectrum sensing, controllers transiting radio frequency signals and Electro-Optical/Infrared (EO/ IR) cameras for visual detection of potential hazards [22]. Sense and avoid technologies are a must and are fundamental part of any equipment that is to be used for BVLOS applications.

\section{RADAR FOR UAS APPLICATIONS}

Radar is a prerequisite for UTM applications for unmanned aircraft. One of the most notable is the Foretem DroneHunter UAV (Fig. 4), which operates a BVLOS as a defence for day and night aerial security and boast as being the first counter drone system that can operate BLOS (Beyond Line of Sight) [23].
The UAV is equipped with a novel piece of hardware called the Fortem TrueView radar model R20 and is based on radar technology used by the US department of defence drone programme. It provides the pilot the ability to detect objects from the air at long ranges to enhance the avoidance of other aircraft, aerial objects and other structures. One of the main additions of this device is the option for complete end to end integration which in turn allows for command and controlled autopilots [24]. It is also proposed that autopilots will be able to execute mission safely even in more crowded spaces due to TrueView Radar as it can detect obstacles in its surroundings with sufficient time to determine the potential of an incident and then in turn stay well clear by manoeuvring to a safe place or to a safe distance.

As well as the Foretem TrueView radar, Sematica Aerospace have developed the Zeus Radar System that has been specifically designed for UAS [25]. The system has been described to enhance situational awareness of any air bound craft entering the nearby airspace by using state of the art solid state radar and advanced signal processing techniques. Solid state radar has the ability to conduct 'sweeps' that can be adjusted in real time by the operator and embodies a range of different signals can be employed for more efficient signal processing [26]. This type of radar can use Doppler radar as well as pulsed radar without the need for extra equipment so that it cannot only see objects within its airspace but also calculate and determine if the objects are moving. Although not a new technique the fact that it has been developed and engineered for UAS means that BVLOS could be one step closer.

Another company that has been working with NASA to develop sense and avoid (SAA) systems is Vigilant Aerospace who have completed successful testing of its new and recently developed FlightHorizon detect and avoidance system [27].

This software provides the operator and autopilots with complete situational awareness, detect and avoid system. By gathering data from various sources such as aviation transponders, ground based radar pulses and air traffic warnings. Vigilant Aerospace also incorporated an exclusive NASA patent software, which forms the backbone of the FlightHorizon product. The invention and patent by Arteaga [28] which is basically an ADS-B system details that traffic information will be included in the transmission and through telemetry communication that is transmitted to a remote ground system. The invention goes

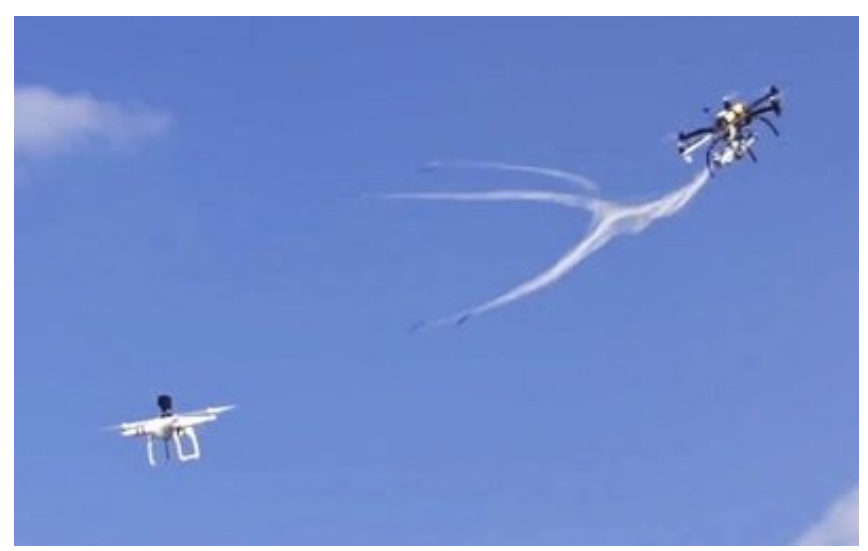

Fig. 4. Foretem DroneHunter in action [23]. 
further to propose the methods for displaying a general layout of aviation traffic information in possibly three or four-dimensional trajectories using an industry standard Earth Browser for heightened situational awareness and an enhanced visual range of possible traffic and obstacles in its flight path. It is also claimed that the novel invention can enable and enhance visual acquisition of traffic and traffic alerts [28].

\section{BVLOS Missions AND ARTIFICIAL INTELLIGENCE (AI)}

In France BVLOS has been permitted since 2012 and the first BVLOS application test was successfully completed for inspecting power lines, by Delair-Tech who flew a UAV for over 30 miles using a $3 \mathrm{G}$ wireless network to guide the drone (Fig. 5). The company were granted a specific flight corridor in which conducted the test flight. Although the flight was conduct via autopilot, two pilots were present at the start and two pilots were present at the landing site. Using the $3 \mathrm{G}$ network allowed for real-time communication from any distance as long as there was $3 \mathrm{G}$ coverage [29].

2017 saw Israel step up its involvement in the BVLOS UAS sector and has recently granted full permission for BVLOS flights. The award was given by the Civil Aviation Authority of Israel (CAAI), to Airobotics who have developed a UAV that can achieve and execute missions safely without the aid of a pilot (Fig. 6). On-board is Airobotics own computer software which also incorporates Artificial Intelligence (AI) which is programmed to make decisions and execute actions that are usually performed by a human pilot [30]. The BVLOS platform is based on three parts. The first component was the UAV, named "Optimus", which is a drone that is capable of flying thirtyminute missions whilst being equipped with a one-kilogram payload. The second component is a completely unmanned, automated airbase from which the UAV can be launched from and also lands on. The third and final piece and the most important is the software and the AI software, which enables operators to use the software easily and manage missions just with one click [31].

This may sound as though the problem that was once facing the drone industry has been solved, however the use of AI itself presents problems of its own. AI itself is a controversial topic for both industry and politics. Keeping $\mathrm{AI}$, or narrow AI, which is purely focused on autonomous drone navigation, at a level that is beneficial for the good of mankind is hotly debated and motivates many research areas although flight safety is always the key element to be

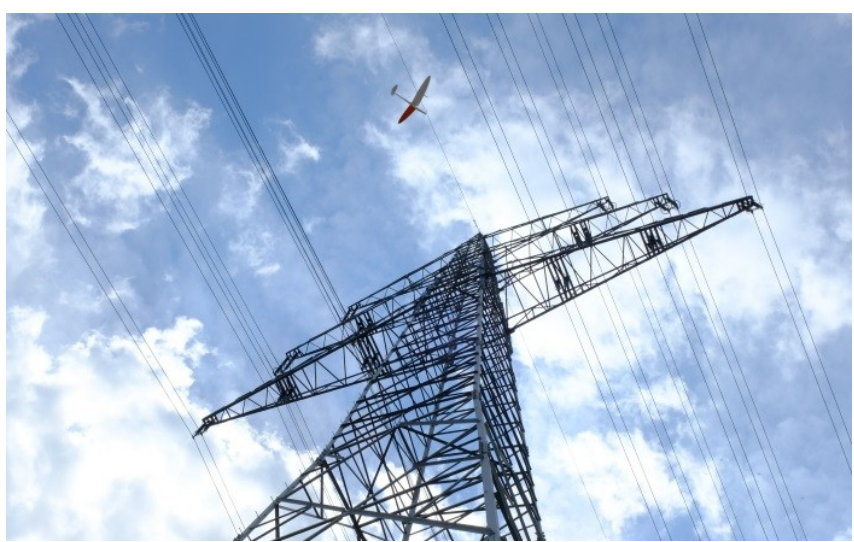

Fig. 5. Delair Tech BVLOS for power line inspection applications [29].

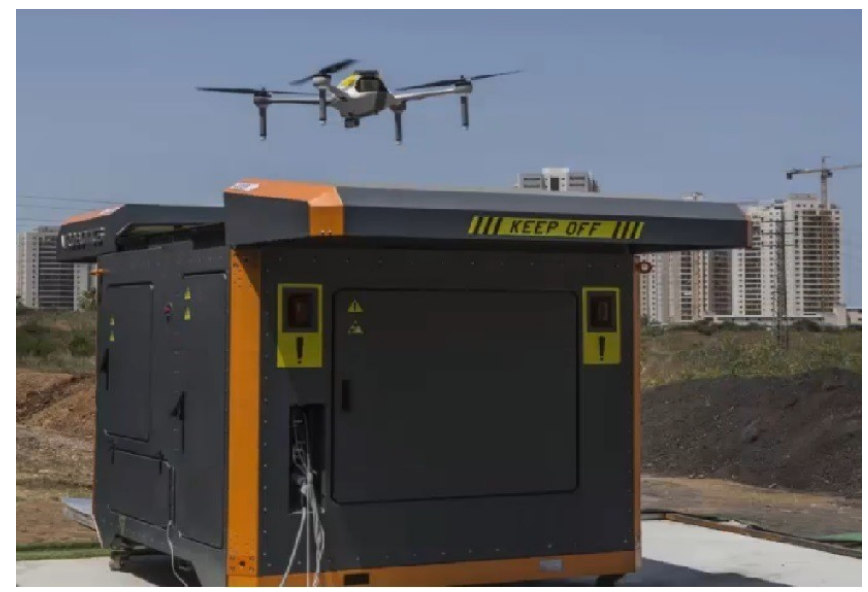

Fig. 6. Airobotics autonomous BVLOS system [33].

considered. The goal for most research is to create general AI that far outgrows the relative conformity if narrow AI [31]. Currently the AI that we are living with are neural networks and machine learning algorithms that are used in everyday common devices [31]. A main concern is for our own preservation as it is feared that AI could at some point become intelligent enough to replace humans and become part of a technological singularity. Indeed this is a situation some may even welcome as they see AI as a panacea for civilisation [32] even though AI might outperform humans at every cognitive task and risks rendering us obsolete [31]. AI will undoubtedly have a major impact on people's lives, but the benefits are undeniable.

\section{UAS SENSOR FUSION}

Sensory communication with any UAS is paramount to operating beyond the pilots' field of vision. A study into potential sensory appliances has been presented by Zhahir et al. [34] and looked at the current development of UAV sense and avoid systems. One possible theory presented as a possible way to achieve safe BVLOS applications, was to equip an UAV with electro-optical sensors combined with radar and infrared sensory capabilities. However, bad weather or overcast and cloudy conditions could affect the performance at object and hazard identification as the sensors rely on good light to be able to work at full capacity. Another possibility discussed was 'sensor fusion', enabling multiple sensory tasks on a UAV platform to be performed simultaneously to enhance hazard detection and minimise flight risks. Ramasamy et al. [35] details a successful test using sensor fusion. The research successfully produced a simulated study of sensor fusion which combines natural inspired sensors and noncooperative sensors. The algorithm that was used by the researchers to achieve this is known as track to track fusion and is based on Boolean decision logic data structure that can evaluate and solve issues such as limited information of the environment or partial loss of transmitted information.

The UAV platform is essential for military applications and its role in conflict and congested zones cannot be underestimated. Small unmanned aircraft embark upon intelligence gathering missions via reconnaissance and surveillance and BVLOS is an essential component. One example of the most state of the Art recognisance UAV that has been developed for BVLOS missions is the military's Black Hornet Nano [36] (Fig. 7). 
This small, compact UAV is fitted with multi-sensory capability and has an integrated video stream data ink where images can be viewed in real time. Part of the UAS ability to perform BVLOS missions is that has the capacity to be programmed with a pre-planned route using GPS and can also be used in FPV with a maximum range of $1.5 \mathrm{~km}$ distance between the operator and the UAV [36]. In comparison larger military UAS rely on satellite communication to operate rather than a direct flowing radio link. The military have further developed a system that can detect other aircraft so that they may be targeted by air to air missiles. The system known as Active Electronically Scanned Array (AESA) radar, which is also known as an active phase array radar, which operates by emitting a pulse signal from a transmitter that in turn is received by an onboard antenna that receives amplified echoes of any objects in the vicinity.

Texas Instruments, in 2016 discussed the benefits of developing a low latency design for video enabled drones [37]. One of the main features that a piloted UAV requires would be an onboard camera as well as a range of other SAA instruments. The needs of the camera are directly linked to the needs of the UAV. A low power consumption rate is necessary so that it does not impact on the UAV's power supply and just as important a low latency data collection design is needed. As with any optical capturing instrument a higher frame rate will lead to lower capture time. This is important when needing to transmit images quickly as the compression and encoding times are greatly reduced. Using industry standard compression format of H.264 will enable this encoding to be initiated quicker with limited visible loss in quality of the image. The research conducted by Texas Instruments proposes to fully utilise low latency and H.264 compression. This is achieved by introducing the concept of "slices" composed of several independently encoded macroblocks which can thus be decoded by itself without any interference of the data capture. This would also naturally decrease the render time of any image. To permit the drone to capture video the camera must be interfaced to the digital processor using one of the dedicated camera interfaces. The feed is then transmitted to a ground control unit using either 2.4 or $5.8 \mathrm{GHz}$ Wi-Fi which in turn will be shown on a display unit for the operator to view the FPV image.

The use of video capturing sensors is a multi-faceted problem, as with any broadcast a reliable transmission signal is a must. As the wireless communication link must

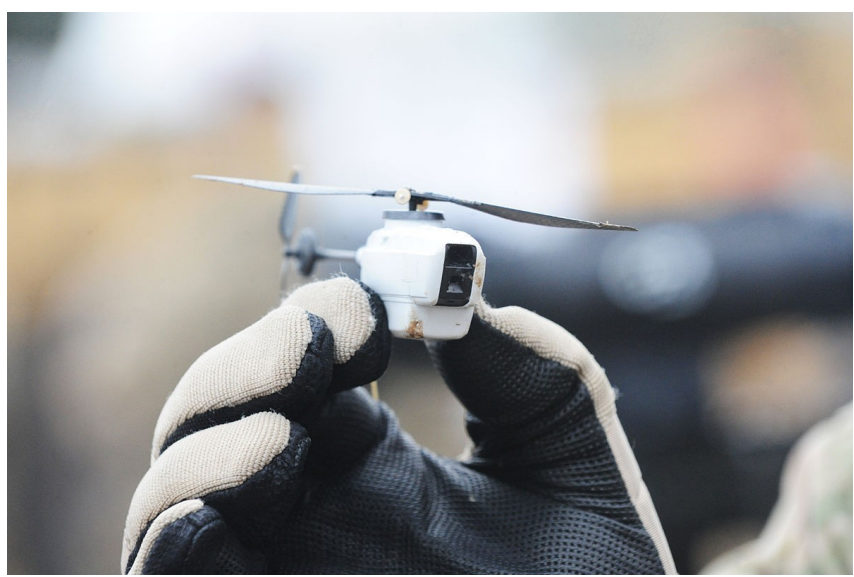

Fig. 7. Black Hornet Nano [38]. be able to cope with long range transmission and reception. The research looks at several ways in which this can be achieved with either antenna diversity, maximum ratio combing (MRC) and Multi-Input and Multi Output (MIMO) and finally rate adaption. This would obviously depend on which wireless network would be available in the area at the time of where the operation is to be flown.

\section{CONCLUSION}

The technology for safe and efficient BVLOS mission completion is already available and seems likely to become common place. There are however, a number of factors which still need to be addressed to ensure the maximum safety for BVLOS operations. The most important of which is UAS communications technology supporting command and control, navigation, surveillance, situation awareness and the integration with Air Traffic Management (ATM) systems for remotely piloted and autonomous aircraft. Development in these technologies and their miniaturisation remains an enabler of future UAS BVLOS capabilities.

The regulations surrounding BVLOS are currently subject to revision as the new European airspace U-Space develops. As BVLOS technology grows and improves so too should the airworthiness regulations to facilitate and guide the industry sector and the deployment of drones in our society.

Autonomous flight capability is not only fundamental to BVLOS operations for UAS but also likely to have a significant impact on the future development of passenger carrying autonomous aircraft. Minimising the Human Factor in aircraft flight has always been a major safety goal and also provides the potential to reduce operational costs. It would therefore appear that the benefits of achieving BVLOS capabilities are likely to outweigh the risks that are currently attributed to an UAS flying beyond an operator's line of sight.

\section{REFERENCES}

[1] Civil Aviation Authority. (2015, March 31). CAP 722 Unmanned Aircraft System Operations in UK Airspace-Guidance. (6th ed.) [Online]. Available: https://publicapps.caa.co.uk/docs/33/CAP\% 20722\%20Sixth\%20Edition\%20March\%202015.pdf

[2] C. Stocker, R. Bennett, F. Nex, M. Gerke, and J. Zevenbergen, "Review of the current state of UAV regulations," Remote Sensing, vol. 9 , no. 5 , article $459,2017$.

[3] European Commission. Press Release. (2017, June 16). Aviation Commission is Taking the European Drone Sector to New Heights. [Online]. Available: http://europa.eu/rapid/press-release_IP-171605 en.pdf

[4] A. Perlman (2017, Feb. 16). "Inside BVLOS, the drone industry's next game-changer", UAV Coach. [Online]. Available: https:// uavcoach.com/inside-bvlos

[5] Imperial War Museums. (2018, Jan. 30). A Brief History of Drones. [Online]. Available: http://www.iwm.org.uk/history/a-brief-historyof-drones

[6] Civil Aviation Authority, CAP 1612 Airspace Change Decision: Beyond Visual Line of Sight Unmanned Aircraft Systems Operations in EG D128 - Everleigh, Gatwick: CAA, 2017.

[7] Defence Infrastructure Organisation. (2017, Dec. 6). Proposal for Beyond Visual Line of Sight Formal (BVLOS) Remotely Piloted Air Systems (RPAS) Operations in EDG 128 - Everleigh. [Online] Available: https:/www.caa.co.uk/uploadedFiles/CAA/Content/ Standard_Content/Commercial_industry/Airspace/ Airspace_change/20171016-FORMAL\%20PROPOSAL $\% 20$ FOR $\%$ 
20BVLOS\%20RPAS\%20OPERATIONS\%20IN\%20D128\% 20EVERLEIGH.pdf

[8] H. Gonzalez-Jorge, J. Martinez-Sanchez, M. Bueno, and P. Arias, "Unmanned aerial systems for civil applications: A review," Drones, vol. 1, no. 1, article 2, 2017.

[9] D. Day, "Drones for transmission infrastructure inspection and mapping improve efficiency," Natural Gas and Electricity, vol. 33, no.12, pp. 7-11, July 2017.

[10] M. Pappota, and R.J. de Boera, "The integration of drones in today's society," Procedia Engineering, vol. 128, pp. 54-63, 2015.

[11] J.-L. Liardon, L. Hostettler, L. Zulliger, K. Kangur, N.G. Shaik, and D.A. Barry, "Lake imaging and monitoring aerial drone," HardwareX, 2018, doi: https://doi.org/10.1016/j.ohx.2017.10.003

[12] V.E. Hovstein, A. Sægrov, and T.A. Johansen, "Experiences with coastal and maritime UAS BLOS operation with phased-array antenna digital payload data link," in Proc. Int. Conf. on Unmanned Aircraft Systems (ICUAS), Orlando, FL, USA, 27-30 May 2014, pp. 261-266.

[13] J. Plaza (2017, March 29). "First commercial drone flight conducted beyond visual line of sight in Canada," Commercial UAV News. [Online]. Available: https://www.expouav.com/news/latest/firstcommercial-drone-flight-conducted-beyond-visual-line-sight-canada

[14] J. Zimmerman (2013, Jan. 17). "ADS-B 101: What it is and why you should care," Air Facts Journal. [Online]. Available: https:// airfactsjournal.com/2013/01/ads-b-101-what-it-is-and-why-youshould-care

[15] A. Costin, and A. Francillon, "Ghost in the Air(Traffic): On insecurity of ADS-B protocol and practical attacks on ADS-B devices," Black Hat, July 2012. [Online]. Available: https:// media.blackhat.com/bh-us-12/Briefings/Costin/ BH_US_12_Costin_Ghosts_In_Air_WP.pdf

[16] V-Tol. (2017, Dec. 21). V-Tol Conducts Advanced BVLOS ADS-B Equipped Flight Operations. [Online]. Available: http://v-tol.com/vtol-conducts-advanced-bvlos-ads-b-equipped-flight-operations

[17] uAvionix. (2018). Ping20S. [Online]. Available: https:// www.uavionix.com/products/ping20s

[18] Lotus Aviation Technology (2018). Electro Optic Gimbal. [Online]. Available: http://www.lotusaviation.com/goshawk_ii_hd.php.

[19] S. Balachandran, C. Munoz, and M. Consiglio, "Implicitly coordinated detect and avoid capability for safe autonomous operation of small UAS," in Proc. 17th AIAA Aviation Technology, Integration, and Operations Conference, Denver, Colorado, 5-9 June 2017, pp.1-10.

[20] M. Johnson, J. Jung, J. Rios, J. Mercer, J. Homola, T. Prevot, D. Mulfinger, and P. Kopardekar, "Flight test evaluation of an unmanned aircraft system traffic management (UTM) concept for multiple beyond-visual-line-of-sight operations," in Proc. 12th USA/ Europe Air Traffic Management Research and Development Seminar, Seattle, WA, USA, 26-30 Jun. 2017, pp. 1-10.

[21] P. Kopardekar, J. Rios, T. Prevot , M. Johnson, J. Jung, and J.E. Robinson III, "Unmanned aircraft system traffic management (UTM) concept of operations," in Proc. 16th AIAA Aviation Technology, Integration, and Operations Conference, Washington, DC, USA, 1317 June 2016, pp. 1-16.

[22] Gryphon Sensors. (2017). What Is Skylight? [Online]. Available: http://gryphonsensors.com/products/\#product-showcase
[23] Fortem Technologies. (2017). Autonomous Counter-UAV System Fortem DroneHunter. [Online]. Available: http:// www.fortemtech.com/dronehunter.html

[24] Fortem Technologies. (2017). Small Long-Range Radar for UAVS. Fortem TrueView. [Online]. Available: http://fortemtech.com/ r20.html

[25] Seamatica Aerospace. (2018). Zeus Radar System. [Online]. Available: http://seamatica.weebly.com/zeus-radar-system.html

[26] T. Burden (2018, Feb. 26). "A new wave of marine radar," West Marine. [Online]. Available: https://www.westmarine.com/ WestAdvisor/New-Radar-Technology

[27] C. Rees (2017, Jan. 30). "New detect-and-avoid system for drones completes BLOS flight tests," Unmanned Systems Technology. [Online]. Available: http:/ www.unmannedsystemstechnology.com/2017/01/vigilant-aerospacecompletes-blos-uas-testing-nasa-flight-research-center

[28] R. Arteaga, "Automatic dependent surveillance broadcast (ADS-B) system for ownership and traffic situational awareness," US Patent 9405005 B1, 2016.

[29] Delair-Tech. Press Release. (2017, June 8). 1st in France: Drone Completes 30 miles BVLOS Flight via $3 G$ Cell Network. [Online]. Available: http://delair.aero/wp-content/uploads/2017/06/PressRelease_RTE_EN-1.pdf

[30] C. Rees (2017, March 31). "Airobotics approved to fly fullyautomated BVLOS drones," Unmanned Systems Technology. [Online]. Available: http:/ www.unmannedsystemstechnology.com/2017/03/airobotics-grantedapproval-fly-fully-automated-commercial-drones

[31] Future of Life Institute. (2017). Benefits and Risks of Artificial Intelligence. [Online]. Available: https://futureoflife.org/background/ benefits-risks-of-artificial-intelligence

[32] D. Galeon (2017, April 15). "Artificial intelligence is only dangerous if humans use it foolishly," Futurism. [Online]. Available: https:// futurism.com/artificial-intelligence-only-dangerous-humans-usefoolishly

[33] G. Bazzolo. (2017, Apr 12). Investment Data: Airobotics from Israel gets the first world license to fly commercial drones fully automated. beBee. [Online]. Available: https://www.bebee.com/producer/ @www-qudron-com/investment-data-airobotics-from-israel-gets-the -first-world-license-to-fly-commercial-drones-fully-automated

[34] A. Zhahir, A. Razali, and M. Mohd Ajir, "Current development of UAV sense and avoid system," IOP Conf. Series: Materials Science and Engineering, vol. 152, article. 012035, 2016.

[35] S. Ramasamy, R. Sabatini, and A. Gardi, "Avionics sensor fusion for small size unmanned aircraft Sense-and-Avoid," in Proc. IEEE Conf. Metrology for Aerospace (MetroAeroSpace), Benevento, Italy, 29-30 May 2014, pp. 271-276.

[36] Aviassist. Commercial Drone Blog. (2017). The Opportunities and Challenges of Flying Beyond Line of Sight (BVLOS). [Online]. Available: https://www.aviassist.com.au/opportunities-challengesflying-drones-beyond-line-sight-bvlos

[37] D. Barrett, and P. Desai (2016). Low-latency Design Considerations for Video-enabled Drones. [Online]. Available: http:/www.ti.com/ lit/wp/spry301/spry301.pdf

[38] Wikipedia. (2017, Oct. 21). Black Hornet Nano. [Online]. Available; https://en.wikipedia.org/wiki/Black_Hornet_Nano 